\title{
Pacific
}

Journal of

Mathematics

\section{THE RELATIVE NIELSEN NUMBER AND BOUNDARY-PRESERVING SURFACE MAPS}

Michael R. Kelly 


\title{
THE RELATIVE NIELSEN NUMBER AND BOUNDARY-PRESERVING SURFACE MAPS
}

\author{
Michael R. Kelly
}

\begin{abstract}
Let $X$ be a compact 2-manifold with nonempty boundary $\partial X$. Given a boundary-preserving map $f:(X, \partial X) \rightarrow(X, \partial X)$ the relative Nielsen number $N_{\partial}(f)$ gives a lower bound for the number of fixed points of $f$. Let $M F_{\partial}[f]$ denote the minimum number of fixed points of all boundary-preserving maps homotopic to $f$ as maps of pairs. This paper continues the study of the difference $M F_{\partial}[f]-$ $N_{\partial}(f)$ for surface maps begun by Brown and Sanderson [BS]. Their results are extended by (i) adding to their list of surfaces for which this difference can be arbitrarily large, and (ii) producing an example of a boundary-preserving map of the pants surface for which the difference is equal to one. This answers a conjecture raised by the authors.
\end{abstract}

1. Introduction. Let $X$ be a compact, connected surface and let $\partial X$ denote its boundary. Given a self-map $f: X \rightarrow X$, following standard notation, let $N(f)$ denote the Nielsen number of $f$ and let $M F[f]$ be the minimum number of fixed points possible for a map homotopic to $f$. It is a classical result that for arbitrary spaces and maps the difference $M F[f]-N(f)$ is always a non-negative integer. For manifolds of dimension greater than two, it is a well-known result of Wecken [W] that $M F[f]=N(f)$. While on the other hand, Jiang $[\mathbf{J}]$ showed that any surface with negative Euler characteristic supports maps for which the difference is strictly positive. Results of the author ([K1], [K2]) have established that for any surface which contains a cohomology injective embedding of the pants surface, the difference can be made arbitrarily large.

In the paper [BS] the authors begin a study of the fixed point behavior for boundary-preserving maps of surfaces. (These are relative maps $f:(X, \partial X) \rightarrow(X, \partial X)$.) It is assumed that all homotopies under consideration are homotopies through boundary-preserving maps. In this setting, the relative Nielsen number as defined by Schirmer [S], and denoted $N_{\partial}(f)$, is a better algebraic invariant than the standard Nielsen number. The analogous minimal number to consider will be denoted $M F_{\partial}[f]$. The intent of their work is to classify all surfaces $X$ (with $\partial X \neq \varnothing$ ) in terms of the following: 
(1) if $N_{\partial}(f)=M F_{\partial}[f]$ for all maps $f$, then $X$ is said to be boundary-Wecken.

(2) if the difference $M F_{\partial}[f]-N_{\partial}(f)$ is bounded, then $X$ is almost boundary-Wecken.

(3) if the difference $M F_{\partial}[f]-N_{\partial}(f)$ can be made arbitrarily large then $X$ is totally non-boundary-Wecken.

REMARK. The term boundary-Wecken will forthwith be abbreviated to bW. Note that (1) is contained in (2), and that (2) and (3) are disjoint. The analogs to (1)-(3) in the non-boundary-preserving setting are referred to as: Wecken, almost-Wecken, and totally non-Wecken. Jiang's result $[\mathbf{J}]$ can then be rephrased as: a surface is Wecken if and only if its euler characteristic is non-negative. An improvement of this is given below in Theorem 1.1.

We now summarize the results of Brown and Sanderson given in [BS]. First, they show that the disk, annulus and the Möbius band are each bW. In [BS; Theorem 3.1] it is established that each surface from the following list is totally non-bW; (i) delete $r \geq 2$ open disks from the torus, (ii) delete $r \geq 1$ open disks from the connected sum of two or more tori, (iii) delete $r \geq 1$ open disks from the connected sum of four or more projective planes. Finally, they show that the pants surface is almost bW, with the bound on the difference being one. They conjecture that this surface is not $\mathrm{bW}$.

The purpose of this paper is to extend the above results of Brown and Sanderson. In Theorem 4.1 a larger class of surfaces (than those mentioned in the list above) are shown to be totally non-bW, and in Theorem 4.3 we show that the pants surface, as conjectured, is not bW. Thus, the notions of $\mathrm{bW}$ and almost $\mathrm{bW}$ are different in the boundarypreserving setting. This is in contrast to the corresponding result for maps of surfaces with boundary as

THEOREM 1.1. If $X$ is a compact, connected surface with non-empty boundary and the Euler characteristic of $X$ is less than zero, then $X$ is totally non-Wecken.

The proof of Theorem 1.1 is a direct consequence of [K2; Theorem 1.1] and Proposition 3.3 of this paper. It is unknown whether or not this extends to closed surfaces as well (the theorem quoted from [K2] only applies to surfaces with boundary). It seems reasonable that the above theorem extends to closed surfaces and thus, the notions of Wecken and almost Wecken would be equivalent. 
The remainder of this paper is concerned with the proofs of Proposition 3.3, and Theorems 4.1 and 4.3. In $\S 2$ the tools from [K1] and [K2] needed to compute the minimal number $M F[\cdot]$ are given. Section 3 shows that all bounded surfaces with fundamental group having rank 2 are totally non-Wecken. This is then used to prove Theorem 4.1. Finally, in $\S 4$, a specific boundary-preserving map $b$ of the pants surface is shown to satisfy $M F_{\partial}[b]-N_{\partial}(b)=1$. As a result of this work, the only surfaces for which this boundary-preserving map classification is unknown are: $S^{2}$ with at least four open disks removed, and projective space with at least two open disks removed.

2. A method for computing $M F[\cdot]$. Let $D$ be a 2-dimensional disk and let $M$ denote the surface obtained by attaching $n$ 1-handles, $H_{1}, \ldots, H_{n}$ to the boundary of $D$. Let $A_{1}, \ldots, A_{2 n}$ be disjoint arcs in $\partial D$ so that $H_{i} \cap D=A_{2 i-1} \cup A_{2 i}, i \in\{1, \ldots, n\}$. Set $A=\bigcup A_{i}$. Choose a basepoint $x_{0}$ in $D$ and let $w_{1}, \ldots, w_{n}$ be oriented based loops in $M$ which intersect in the point $x_{0}$, and $w_{i} \cap H_{j}$ is an arc if $i=j$, and empty otherwise. To remove any ambiguity in the indexing of the components of $A$, it is assumed that when the oriented loop $w_{i}$ is traversed, that $A_{2 i-1}$ is traversed before $A_{2 i}$. Let $W=\bigcup_{i=1}^{n} w_{i}$ and let $s: M \rightarrow W$ be a strong deformation retraction such that $s^{-1}(x)$ is a proper arc in $H_{i}$ for each $x \in\left(W \cap H_{i}\right)$.

By abuse of notation we let the based loops $w_{1}, \ldots, w_{n}$ also denote elements in the fundamental group of $M$, and hence $\pi_{1}\left(M, x_{0}\right)$ is the free group generated by these $n$ loops. Given an endomorphism $\phi: \pi_{1}(M) \rightarrow \pi_{1}(M)$, define a map $\hat{\phi}: W \rightarrow W$ such that $\hat{\phi}(W \cap D)$ $=x_{0}$, and $\hat{\phi}\left(W \cap H_{i}\right)$ traces out the word in $W$ corresponding to $\phi\left(w_{i}\right)$. Define $f_{\phi}: M \rightarrow M$ by $f_{\phi}=i \circ \hat{\phi} \circ s$ where $i: W \rightarrow M$ denotes inclusion. Notice that $f_{\phi}^{-1}(A)$ consists of proper arcs in $M$ (all contained in $\bigcup H_{i}$ ), and each of $H_{i}$ contains $2 m_{i}$ arcs, where $m_{i}$ is the length of the reduced word $\phi\left(w_{i}\right)$.

For the following we assume that given $g: M \rightarrow M$, then $g(M) \subset$ int $M$ and that $g^{-1}(A)$ is a 1-dimensional proper submanifold of $M$. With $\mathscr{C}$ denoting the set of components of $g^{-1}(A)$, define a function $\mu: \mathscr{C} \rightarrow\{1, \ldots, 2 n\}$ so that $\mu(C)=k$ iff $g(C) \subset A_{k}$ for each $C \in \mathscr{C}$. The function $\mu$ extends naturally to subsets of components of $g^{-1}(A)$. Notice that, by general position, there exists a map having the minimal number of fixed points, which satisfies the condition above. Further, we may assume that $g^{-1}(A)$ has the property that for each open set $\mathscr{O}$ in $M$ that meets $g^{-1}(A), g(\mathscr{O})$ is not contained in the closure of a component of $M \backslash A$ (see [K1; Lemma 3.2]). Other ways 
to simplify maps having the minimal number of fixed points are given in the following two lemmas. A proper arc in $M$ is called a boundary curve if it is isotopic (rel endpoints) to an arc which is disjoint from $W$.

LEMMA 2.1. Given a map $f: M \rightarrow M$ there is a map $g$ homotopic to $f$ such that \#Fix $(g)=M F[f]$ and $g^{-1}(A)$ is a 1-dimensional proper submanifold of $M$. In addition, there is a map $h$, homotopic to $g$, also having $M F[f]$ fixed points and such that the collection of curves $h^{-1}(A)$ is properly isotopic to the collection obtained by remov: ing all of the boundary curves and inessential simple closed curves from $g^{-1}(A)$.

Proof. That $g^{-1}(A)$ is a proper submanifold and the removal of the inessential simple closed curves is given in [K1; Lemma 3.2, Corollary 3.7]. The following construction for removing boundary curves, of which a variation will also be used in the proof of Proposition 3.2, is referred to as the "collar construction."

Suppose that $\beta$ is a boundary curve in $g^{-1}(A)$. Let $C \equiv S^{1} \times I$ be an annulus and form a surface $M^{\prime}$ by identifying the boundary component of $M$ containing the endpoints of $\beta$ with $S^{1} \times\{0\}$. Let $A_{i}^{\prime}=A_{i} \cup\left(\left(\partial A_{i} \cap c\right) \times I\right)$. Since $\beta$ is a boundary curve there is a unique arc $\beta_{0}$ contained in $\partial M$ such that $\beta \cup \beta_{0}$ forms an inessential simple closed curve. Let $\beta^{\prime}$ be a proper arc in $C$ chosen so that $\beta_{0} \cup \beta^{\prime}$ forms an inessential simple closed curve. Then clearly, $\beta \cup \beta^{\prime}$ forms an inessential simple closed curve. Moreover, if $\beta_{1}$ and $\beta_{2}$ are two such curves, then the new curves constructed in $M^{\prime}$ can be taken to be disjoint. Apply this construction to all of the boundary curves; to all the other arcs in $M$ extend straight outwards to $\partial M^{\prime}$. Let $\Gamma$ denote the new collection of curves in $M^{\prime}$, and define a map $G: M^{\prime} \rightarrow M^{\prime}$ extending $g$ so that $G^{-1}\left(A^{\prime}\right)=\Gamma$ and $G(C) \subset$ int $M$. Then clearly, \#Fix $(G)=\# \operatorname{Fix}(g)$. Finally, remove these newly formed inessential simple closed curves to obtain $h$, noting that $M^{\prime}$ is homeomorphic to $M$.

LEMMA 2.2. Suppose $\delta$ and $\eta$ are curves in $g^{-1}(A)$ with $\mu(\delta)=$ $\mu(\eta)$, and $\lambda$ is an arc in $M$ such that $\partial \lambda$ meets each of $\delta, \eta$ and int $\lambda \cap g^{-1}(A)=\varnothing$. Let $\left(U, U_{0}\right)$ be a closed regular neighborhood of $(\lambda, \partial \lambda)$ in $\left(M_{0}, g^{-1}(A)\right)$ where $M_{0}$ is the closure of the component of $M \backslash g^{-1}(A)$ which contains int $\lambda$. If there is a disk $X$ which contains both $g(U)$ and the subarc of $A_{\mu(\delta)}$ containing $g(\partial \lambda)$ with $X \cap U=\varnothing$, 
then there is a map $h$ homotopic to $g$ with support on a neighborhood of $U$ such that $\operatorname{Fix}(h)=\operatorname{Fix}(g)$ and

$$
h^{-1}(A)=\left(g^{-1}(A) \cup \partial U\right) \backslash \operatorname{int} U_{0} .
$$

Proof. Let $V$ be a regular neighborhood of $U$ in $M$ that meets $g^{-1}(A)$ in two arcs, and such that $V \cap X=\varnothing$ and $g(V) \subset X$. Define $h$ so that it agrees with $g$ on the set $(M \backslash V) \cup\left(g^{-1}(A) \backslash U_{0}\right)$ and so that $h\left(\partial U \backslash U_{0}\right) \subset A_{\mu(\delta)}$. Extend $h$ to the rest of $V$ by mapping each of the three remaining disks into $X \backslash A$. The result follows as $h(V) \cap V=\varnothing$.

Our mechanism for estimating $M F[\cdot]$ is Theorem 2.3 given below which is taken from Theorem 3.1 of [K2]. To apply this theorem we will need some terminology regarding self-maps of $M$. An arc or a simple closed curve, $\gamma$, in $M$ is said to be taut if $\partial \gamma \cap A=\varnothing$, int $\gamma$ meets $W \cup A$ transversally in a finite number of points, $A \cup \gamma$ does not contain any inessential simple closed curves, and for each $i$, int $\gamma \cup\left(w_{i} \backslash x_{0}\right)$ does not contain any simple closed curves. This last condition keeps the geometric intersection of $\gamma$ and $W$ minimal up to isotopy $(\operatorname{rel} \partial)$ in $M \backslash x_{0}$. A map $f: M \rightarrow M$ is taut if each component of $f^{-1}(A)$ is a taut proper arc or a taut simple closed curve, $\operatorname{Fix}(f) \cap A=\varnothing$ and $f(M) \subset W$. Let $C$ denote the closure of a component of $M \backslash\left(f^{-1}(A) \cup A\right)$. We say that $C$ is a critical region for $f$ if $f(C)$ meets the same component of $M \backslash A$ as does $C$. We denote the topological fixed point index of $f$ restricted by $C$ by index $(C)$. A critical region is exceptional if it meets at least three components of $A$. Note that up to homotopy, we may assume that each critical region contains at most one fixed point depending on its fixed point index. For what follows, we assume that each exceptional critical region contains exactly one fixed point even if its index is zero.

A taut arc $\alpha$ contained in the interior of $M$ is a simple merging arc for $f$ if it satisfies the following conditions.

(a1) $\partial \alpha \subset \operatorname{Fix}(f)$.

(a2) $\alpha$ meets $f^{-1}(A)$ transversally and for each $i, \alpha \cup A_{i}$ does not contain any loops which bound disks in $M$.

(a3) If $\alpha_{0}$ is a component of $(\alpha \backslash A)$, and $\tau$ is a component of $\left(f^{-1}(A) \backslash A\right)$, with $\tau \cap \alpha_{0}$ being nonempty, then $\alpha_{0}$ and $\tau$ meet in exactly one point and their closures intersect at least three components of $A$.

(a4) $f(\alpha)$ meets the same components of $A$, in the same order, as $\alpha$. 
This definition is slightly different than the one given in [K2]. The above was obtained by dropping the more general notion of a (nonsimple) merging sequence, and at the same time including condition (a4) which was a property that simple merging arcs had (in that paper). We will see that $(\mathrm{a} 4)$ is very useful when it comes to applying Theorem 2.3. Note that (a4) implies that the endpoints of a simple merging arc are Nielsen equivalent.

Suppose that $\alpha$ is a simple merging arc for $f$. We define a new map $g$ as follows: condition (a4) gives a natural pairing between the finite sets $\alpha \cap A$ and $\alpha \cap f^{-1}(A)$. Given a point $p$ in the latter, let $p^{\prime}$ be its paired point in the former, and let $\alpha_{p}$ be the subarc of $\alpha$ joining the two. Let $C_{p}$ be a disk which contains $\alpha_{p}$ and meets both of $A$ and $f^{-1}(A)$ in a number of arcs, one for each point in $\alpha_{p} \cap\left(A \cup f^{-1}(A)\right)$. Let $\gamma_{p}$ be the arc in $C_{p} \cap f^{-1}(A)$ containing $p$, let $B_{p}$ be the component of $C_{p} \backslash \gamma_{p}$ which contains $p^{\prime}$, and let $\zeta_{p}$ denote the arc $\partial C_{p} \cap B_{p}$. Replace $\gamma_{p}$ with $\zeta_{p}$ to obtain a new curve. Loosely, a "finger-push" of $\gamma_{p}$ along $\alpha_{p}$ has been performed to obtain this new curve. If $q$ is another point in $\alpha \cap f^{-1}(A)$ the disk $C_{q}$ is chosen as above with the additional requirement that the arc $\zeta_{q}$ is kept disjoint from $\zeta_{p}$. Repeating this for each point in $\alpha \cap f^{-1}(A)$ we obtain a new collection of curves which is denoted by $\Gamma$. Then $f$ is homotopic to a map $g$, by a homotopy which is the identity outside a neighborhood of $\alpha$, such that $\Gamma=g^{-1}(A)$. We say that $g$ is obtained from $f$ by merging along $\alpha$, and write $f \stackrel{\alpha}{\rightarrow} g$.

In general, $f \stackrel{\Lambda}{\rightarrow} g$ where $\Lambda=\left(\lambda_{1}, \ldots, \lambda_{r}\right)$ means there exist maps $f_{i}: M \rightarrow M$ with $f_{0}=f, f_{r}=g$, and $f_{i-1} \stackrel{\lambda_{i}}{\rightarrow} f_{i}$. If $\operatorname{int}\left(\lambda_{i}\right) \operatorname{nint}\left(\lambda_{j}\right)=$ $\varnothing$ when $i \neq j$, we call $\Lambda$ a merging sequence for $f$ and note that $\Lambda$ is an ordered sequence as $\partial \lambda_{i}$ may not be in $\operatorname{Fix}\left(f_{i-2}\right)$.

Finally, in order to state Theorem 2.3 an additional map defined in [K2] is needed. Starting with a map $f: M \rightarrow M$ (say as the end of a merging sequence) there is an associated map, denoted $f_{\min }$, which depends only on $f$. The actual definition of this map is omitted here as it is not needed for our computations. Instead we state in Lemma 2.4 the one property of $f_{\min }$ that will be used. This lemma by-passes the computation of $f_{\min }$ by giving sufficient conditions as to when a given fixed point of $f$ will persist as a fixed point of $f_{\min }$. The proof of this lemma follows immediately from the definition of the $\operatorname{map} f_{\min }$.

THEOREM 2.3. Given an endomorphism $\phi$ of $\pi_{1}\left(M, x_{0}\right)$ there exist a taut map $g: M \rightarrow M$, homotopic to $f_{\phi}$, and a simple merging 
sequence $g \stackrel{\Lambda}{\rightarrow} f$ such that \#Fix $\left(f_{\min }\right)=M F\left[f_{\phi}\right]$. Moreover, if $h$ is a map homotopic to $f_{\phi}$ having $M F\left[f_{\phi}\right]$ fixed points and such that $h^{-1}(A)$ is a 1-dimensional proper submanifold of $M$, then $g$ may be chosen so that $g^{-1}(A)$ is isotopic, by an isotopy which is the identity on $\partial M$, to $h^{-1}(A)$.

LEMMA 2.4. Let $f$ be a taut map and suppose $C$ is a critical region for $f$. If for each arc $\zeta$ in $\partial C \cap f^{-1}(A)$ it follows that $A_{\mu(\zeta)}$ is disjoint from $\zeta \cap A$, then there is a unique critical region $C_{\min }$ for $f_{\min }$ such that

$$
\operatorname{index}\left(C_{\min }, f_{\min }\right)=\operatorname{index}(C, f) .
$$

Moreover, if $C$ and $C^{\prime}$ are two such regions $\left(C \neq C^{\prime}\right)$, then $C_{\min }$ and $C_{\min }^{\prime}$ are distinct.

3. Bounded surfaces with $\operatorname{rank}\left(\pi_{1}\right)=2$. In this section we assume that $M=D \cup H_{1} \cup H_{2}$ where $D, H_{1}, H_{2}$ are as defined as $\S 2$. By convention, we assume that $M \backslash H_{1}$ is orientable. Also, the only endomorphisms of $\pi(M)$ which will be considered are those of the form: $\phi\left(w_{1}\right)$ is a nontrivial cyclically reduced word and $\phi\left(w_{2}\right)=1$.

LEMMA 3.1. With $M$ and $\phi$ as above, there is a map $h$, homotopic to $f_{\phi}$, having $M F\left[f_{\phi}\right]$ fixed points and such that each component of $h^{-1}(A)$ is isotopic to one of the following. (i) the simple closed curve $w_{2}$, (ii) a simple closed curve which is isotopic to a boundary component of $M$, (iii) an arc which is disjoint from $A \cup w_{2}$.

Proof. Let $h$ be a map satisfying the conclusions of Lemma 2.1 and suppose that $\beta$ is a simple closed curve contained in $h^{-1}(A)$. Then $[\beta] \in \operatorname{ker} \phi$, where $[\beta]$ denotes the free homotopy class of loops containing $\beta$. We consider the various surfaces in question. For the pants surface, that $\beta$ is isotopic to $w_{2}$ is clear. The same holds when $M$ is a punctured Möbius band.

Now, if $M$ is the punctured torus, let $\widehat{M}$ denote the torus obtained by attaching a disk to $\partial M$. Let $\hat{\beta}$ be the inclusion of $\beta$ in $\widehat{M}$ and let $\hat{\phi}$ be the induced endomorphism. Then $\hat{\beta}$ is a $(p, q)$ curve where $p, q$ are relatively prime integers. A direct calculation shows that $[\hat{\beta}] \in \operatorname{ker} \hat{\phi}$ only when $\beta$ is isotopic to either $w_{2}$ or $\partial M$. Finally, the argument for the punctured Klein bottle is the same, again yielding either $w_{2}$ or $\partial M$. 
For proper arcs in $M$, first consider a map $g$ homotopic to $h$ such that $g^{-1}(A)$ is properly isotopic to $h^{-1}(A)$, and each of its components has minimal intersection with $A \cup w_{2}$. We first consider the case when $M$ is either a punctured torus or punctured Klein bottle. Suppose $\zeta$ is a proper arc in $g^{-1}(A)$ which intersects $A$. By capping off $\partial M$ with a disk and extending $\zeta$ to a simple closed curve $\zeta^{\prime}$ by adding an arc in this disk, it is easy to see that $\zeta$ must traverse $w_{2}$ as well.

At this point we need to reduce. Consider the finite set $g^{-1}(A) \cap$ $w_{2}$. Since $\phi\left(w_{2}\right)=1$, there are adjacent points $p_{1}, p_{2}$ in this set with $\mu\left(p_{1}\right)=\mu\left(p_{2}\right)$. Moreover, since this set lies on a circle, there is another pair $q_{1}, q_{2}$ of adjacent points with $\mu\left(q_{1}\right)=\mu\left(q_{2}\right)$. Let $X_{p}$ and $X_{q}$ denote the components of $M \backslash g^{-1}(A)$ which have $\left\{p_{1}, p_{2}\right\}$ and $\left\{q_{1}, q_{2}\right\}$, respectively, in the boundaries of their closures. Let $n$ be the number of proper arcs in $g^{-1}(A)$ which are contained in the boundary of the closure of $X_{p}$. First notice that $n>1$, for if $\zeta$ was a proper arc containing both of $p_{1}, p_{2}$ then a small deleted neighborhood of $\zeta$ would map into a single component of $M \backslash A$. Also, as $g^{-1}(A)$ does not contain any boundary curves it must be that $n \leq 4$. In case $n$ is either 2 or 3 , there is an arc in $\partial M \cap \operatorname{cl}\left(X_{p}\right)$ which joins an endpoint of the arc containing $p_{1}$ to an endpoint of the arc containing $p_{2}$. In case $n$ is 4 , then $M \backslash X_{p}$ must have two components; each bounded by 2 arcs from $\partial M$ and two arcs from $g^{-1}(A)$. Note that in this last case the closure of $X_{q}$ contains only 2 arcs from $g^{-1}(A)$.

Let $X=\operatorname{cl}\left(X_{p}\right)$ when $n<4, \operatorname{cl}\left(X_{q}\right)$ otherwise. Then there is an arc in $\partial M \cap X$ which joins the paired curves in the boundary of $X$. Let $Y$ denote the closure of the component of $h^{-1}(A)$ corresponding to $X$ an let $\eta_{1}, \eta_{2}$ be corresponding paired curves. Let $\lambda$ be an arc in $Y$ going from $\eta_{1}$ to $\eta_{2}$ and chosen close enough to $\partial M$ so that the hypotheses of Lemma 2.2 are satisfied. Apply the lemma to replace $\eta_{1} \cup \eta_{2}$ with two other arcs, noting that for the new corresponding map $g$ we will have reduced the cardinality of $g^{-1}(A) \cap w_{2}$. Remove any boundary curves and repeat until all curves in $g^{-1}(A)$ have been removed from $w_{2}$.

If $M$ denotes the pants surface, there is only one type of arc which meets $A$. This arc traverses $H_{2}$ once and has endpoints in the boundary component of $M$ which does not intersect $H_{2}$. The endpoints of an innermost such curve are also the endpoints of an arc in $\partial M$ which misses all other components of $g^{-1}(A)$. Lemma 2.2 can now be used 
as above to replace this arc with a boundary curve and a simple closed curve isotopic to $w_{2}$. On the other hand, if all of the proper arcs are disjoint from $A$, Lemma 2.2 can be applied to make them disjoint from $w_{2}$ as well. Finally, for the punctured Möbius band all arcs are disjoint from $A$ and by the same argument, can be made disjoint from $w_{2}$.

The above lemma says that very few types of curves need be considered when searching for a fixed point minimizing map. The next result takes advantage of this to further reduce the possibilities. For convenience, the following terminology will be adopted throughout the rest of this paper. Any curve properly isotopic to $A_{1}$ will be referred to as an $\alpha_{1}$-curve. An arc which is properly isotopic to an arc disjoint from $A$ and is not an $\alpha_{1}$-curve will be called an $\alpha_{0}$-curve. Similarly, a $w_{i}$-curve, $i \in\{1,2\}$, is a simple closed curve isotopic to $w_{i}$.

Proposition 3.2. Let $M$ and $\phi$ be as in Lemma 3.1. Then there is a map $\hat{f}$ homotopic to $f_{\phi}$ such that, $\# \operatorname{Fix}(\hat{f})=M F\left[f_{\phi}\right]$, and $\hat{f}^{-1}(A)$ consists of either $2 l w_{2}$-curves if $M$ is a punctured torus or punctured Klein bottle, or $2 l$ proper arcs, each of which is isotopic rel $\partial M$ to an arc contained in $H_{1}$, otherwise.

Proof. Let $f$ be a map homotopic to $f_{\phi}$ which has $M F\left[f_{\phi}\right]$ fixed points and satisfies the conclusion of Lemma 3.1. Let's first consider the case when $M$ denotes either the punctured torus or punctured Klein bottle. Note that in this case each $\alpha_{0}$-curve meets $w_{2}$ algebraically in one point and so none occur. Thus, $f^{-1}(A)$ consists of $\alpha_{1}$ - and $w_{2}$-curves. Now apply the collar construction, this time adding to each $\alpha_{1}$-curve an arc in $C$ so that the union forms a simple closed curve isotopic to $w_{2}$. Call the resulting map $g$. It has the property that each component of $g^{-1}(A)$ is isotopic to $w_{2}$.

Since $g_{\#}\left(w_{1}\right)$ is conjugate to $\phi\left(w_{1}\right)$, there must be at least $2 l$ of these curves. If there are more than $2 l$, then there must be two curves having the same $\mu$-values, which form the boundary of the closure of the component $T$ of $M \backslash g^{-1}(A)$. Clearly, the closure of $T$ is an annulus. Since each of $A_{3}$ and $A_{4}$ have nonzero intersection number with $w_{2}, T \cap A$ must contain arcs $\lambda_{3} \subset A_{3}$ and $\lambda_{4} \subset A_{4}$ whose boundaries meet both components of the boundary of $\mathrm{cl}(T)$. As $g(T)$ is contained in $M \backslash A$ and $g(\partial T)$ meets only one component of $A$, it follows that $g\left(\lambda_{3} \cup \lambda_{4}\right)$ meets only one component of $A$. Hence, one of $\lambda_{3} \cap g\left(\lambda_{3}\right)$ or $\lambda_{4} \cap g\left(\lambda_{4}\right)$ must be empty. The hypotheses of Lemma 
2.2 are satisfied so $\partial T$ can be replaced by a single inessential simple closed curve which, by Lemma 2.1 , is removed. After removing all excess pairs in this manner we have the desired map $\hat{f}$.

If $M$ is either the pants surface or the punctured Möbius band proceed as follows. Starting with the $\alpha_{0}$-curve nearest $\mathrm{H}_{2}$ (if any exist), connect points near the endpoints with an arc, passing through the handle $\mathrm{H}_{2}$, chosen close to $\partial \mathrm{M}$. Apply Lemma 2.2, so as to replace the curve with a boundary curve and a $w_{2}$-curve. Remove the boundary curve and repeat until all $\alpha_{0}$-curves are gone. Now apply the collar construction by attaching a collar to the boundary component which is isotopic to $w_{2}$. By adding $w_{2}$-curves to $C$ with $\mu$-values assigned so that they pair up with the $w_{2}$-curves already present, we apply the "annulus" construction as in the previous case to remove all of the $w_{2}$-curves. As excess $\alpha_{1}$-curves must be paired, Lemma 2.2 can be used to convert each pair into a pair of boundary curves which are then removed. Finally, to get the $\alpha_{1}$-curves isotopic rel $\partial M$, add collars to the boundary components meeting $H_{1}$ and extend each curve by adding two arcs, one in each collar.

We are now in a position to compute $M F[\cdot]$ for maps whose induced endomorphism of the fundamental group sends $w_{2}$ to the trivial word. To do so, for each positive integer $n$, consider the endomorphism $\phi(n)$, of $\pi_{1}\left(M, x_{0}\right)$, defined by sending

$$
\begin{aligned}
& w_{1} \mapsto w_{1}^{-n} w_{2} w_{1}^{n} w_{2}, \\
& w_{2} \mapsto 1 .
\end{aligned}
$$

By a straightforward computation it can be shown that, for each $n$, the Nielsen number of $f_{\phi(n)}$ is equal to 1 .

Proposition 3.3. With $\phi(n)$ defined as above, $M F\left[f_{\phi(n)}\right] \geq 2 n-5$.

Proof. Let $h$ be a map homotopic to $f_{\phi(n)}$ having the minimal number of fixed points. By Proposition 3.2 and Theorem 2.3, we can assume that there is a merging sequence $g \stackrel{\Lambda}{\rightarrow} f$ with $h=f_{\min }$, and such that $g^{-1}(A)$ consists of $4 n+4$ taut curves; $w_{2}$-curves if $m$ is either a punctured torus or a punctured Klein bottle, $\alpha_{1}$-curves in $H_{1}$ otherwise. The remainder of the proof is summarized as follows: we show that there are at least $2 n-5$ critical regions for $g$ which (1) have non-zero index and (2) are disjoint from $\Lambda$. Hence they are critical regions for $f$. Finally, we appeal to Lemma 2.4 to show that each of these regions corresponds to a fixed point of $h$. 
For the following, let $X=D$, if $M$ is a punctured torus or a punctured Klein bottle, otherwise let $X=H_{1}$. Let $R_{1}, \ldots, R_{r}$ denote the critical regions for $g$ which are contained in $X$ such that $R_{i}$ is bounded by curves $c_{i, 1}, c_{i, 2}$ with $\mu\left(c_{i, j}\right)=j$. Assume that these regions are indexed by their order along $w_{1}$ with $R_{1}$ being nearest $A_{1}$. A direct index calculation shows that index $\left(R_{i}\right)= \pm 1$. The number of such regions can be estimated as follows. Each curve in $g^{-1}(A)$ with $\mu=1$ determines a unique critical region in $X$. At most two are bounded by a second curve with $\mu \in\{3,4\}$, and at most one of these critical regions meets exactly one curve in $g^{-1}(A)$. All others are of the desired type and hence, $r \geq 2 n-3$.

The proof is completed by showing that, for $1<i<r, R_{i}$ is disjoint from $\Lambda$, together with the observation that each $R_{i}$ satisfies the hypothesis of Lemma 2.4. Let $\lambda_{1}, \ldots, \lambda_{m}$ denote the merging arcs (in order) in $\Lambda$ and first consider $g \stackrel{\lambda_{1}}{\rightarrow} g_{1}$. If $\lambda_{1} \cap R_{i} \neq \varnothing$, then $\lambda_{1}$ meets at least one of $c_{i, j}$ and so $g\left(\lambda_{1}\right)$ meets $A_{j}$. By condition (a4), we have that $\lambda_{1}$ crosses $A_{j}$ as well, implying that $\lambda_{1}$ traverses either $R_{1}$ or $R_{r}$. As a result $g\left(\lambda_{1}\right)$, and hence $\lambda_{1}$ as well, contains a subarc in $X$ which goes from $A_{1}$ to $A_{2}$. This subarc of $\lambda_{1}$ meets every component of $g^{-1}(A)$ and so its image under $g$ traces out a cyclic permutation of $\phi(n)\left(w_{1}\right)$. As $\lambda_{1}$ must also contain a subarc traversing $A$ according to this permutation we obtain, for any $n \geq 3$, a contradiction to Lemma 3.4 given below.

As a consequence of the above argument, $\lambda_{1}$ meets at most one curve $\tau$ in $g^{-1}(A)$ which has $\mu(\tau) \in\{1,2\}$. Also, $\tau$ does not lie on the boundary of any of $R_{2}, \ldots, R_{r-1}$. After merging along $\lambda_{1}, \tau$ is deformed to a curve in $g_{1}^{-1}(A)$ which only differs from $\tau$ by a "fingerpush" across $A_{\mu(\tau)}$. This allows us to repeat the same argument to establish that each of $\lambda_{2}, \ldots, \lambda_{m}$ is disjoint from $R_{i}$ when $1<i<r$.

LemMA 3.4. Let $g: M \rightarrow M$ be as in the proof of Proposition 3.3. If $\sigma$ is an arc in $M$ which traverses $A$ in either the order $A_{1}, A_{2}, A_{1}, A_{3}, A_{4}, A_{1}, A_{2}, A_{1}$ of the order $A_{2}, A_{1}, A_{2}, A_{3}, A_{4}$, $A_{2}, A_{1}, A_{2}$, then $\sigma$ cannot be a subarc of a merging arc for $g$.

Proof. Let $\sigma^{\prime}$ denote the subarc of $\sigma$ going from $A_{3}$ to $A_{4}$ in $H_{2}$. From condition (a2), we see that $\sigma^{\prime}$ is disjoint from $g^{-1}(A)$. By the definition of $\sigma$ and the structure of the collection of curves $g^{-1}(A)$, it is easy to see that each component of $\sigma \backslash \sigma^{\prime}$ must intersect $g^{-1}(A)$. Let $\sigma_{0}$ be the maximal subarc of $\sigma$ containing $\sigma^{\prime}$, and having interior disjoint from $g^{-1}(A)$. 
Now, if $\sigma$ were a merging arc, then both endpoints of $\sigma_{0}$ would be contained in the same component of $g^{-1}(A)$. Hence, $g\left(\sigma_{0}\right)$ has endpoints in some $A_{i}$ and interior disjoint from $A$, and thus $\sigma$ would contain a subarc with the same property. But this contradicts condition (a2) of the definition of merging arc.

4. Boundary-preserving maps. In this section we give two distinct results concerning boundary-preserving maps of surfaces. The first extends the known family of totally non-bW surfaces. The second, Theorem 4.3, shows that the pants surface is exceptional in regards to boundary-preserving maps in that it is the only known surface which is almost bW but not bW.

THEOREM 4.1. Let $r$ be a positive integer and let $Y$ be a connected, closed surface other than the two sphere or the projective plane. If $X$ denotes the surface obtained by deleting $r$ open discs from $Y$, then $X$ is totally non- $b W$.

Proof. Observe that for each $n$, the map $\phi(n)$ given in $\S 3$ is homotopic to a boundary-preserving map if $M$ is either a punctured torus or a punctured Klein bottle. Thus, as a result of Proposition 3.3, these two spaces are totally non-bW.

Now, if $Y$ is anything other than the connected sum of either two or three projective planes, then Theorem 3.1 of [BS] says that $X$ is totally non-bW. Their proof is based on the fact that there are two simple closed curves $\alpha, \beta$ embedded nicely (their union is injective on homology) in $X$ so that a regular neighborhood of $\alpha \cup \beta$ in $X$ is the pants surface-together with the fact that the pants surface is totally non-Wecken. Proposition 3.3 can be used to generalize that proof. Namely, if $\alpha \cup \beta$ has a regular neighborhood which is a totally nonWecken surface with fundamental group having rank two, then their argument goes through. If $Y$ is the connected sum of two projective planes, then $\alpha$ and $\beta$ can be chosen so that their regular neighborhood is a punctured Klein bottle. For three projective planes, the resulting surface is a punctured torus.

Suppose $f:(X, \partial X) \rightarrow(X, \partial X)$ is given where $X$ is a compact surface with nonempty boundary. Analogous to Lemma 2.1, there is a map $h:(X, \partial X) \rightarrow(X, \partial X)$ having $M F_{\partial}[f]$ fixed points with $h^{-1}(A)$ being a proper 1-dimensional submanifold of $X$. In addition, we may also assume that no inessential simple closed curves appear. 
Also, for the following, we assume that for all boundary-preserving maps under consideration, the map $f \mid \partial X$ has the minimal number of fixed points possible. This is determined by the degree of the restricted mapping on each invariant component.

By slightly altering the definition of a merging arc to allow for its endpoints to be fixed points in $\partial X$, the proof of [K2; Theorem 3.1] goes through unchanged (except, of course, making sure that all maps are boundary-preserving) in the boundary-preserving setting to give us the following

THEOREM 4.2. Let $f:(X, \partial X) \rightarrow(X, \partial X)$ be such that $f \mid \partial X$ has the minimal number of fixed points. Then there is a taut map $g$, homotopic to $f$, and a simple merging sequence $g \stackrel{\Lambda}{\rightarrow} \hat{g}$ such that \#Fix $\left(\hat{g}_{\text {min }}\right)=M F_{\partial}[f]$. Moreover, if $h$ is a map homotopic to $f$ having $M F_{\partial}[f]$ fixed points and such that $h^{-1}(A)$ is a 1-dimensional proper submanifold of $M$, then $g$ may be chosen so that $g^{-1}(A)$ is isotopic, by an isotopy which is the identity on $\partial M$, to $h^{-1}(A)$.

Let $P$ denote the pants surface with a handle structure as given in $\S \S 2$ and 3. Without loss we assume that $w_{1}$ and $w_{2}$ are chosen so that the loop $w_{1} w_{2}$ is homotopic to a simple closed curve in $P$. Let $C_{0}, C_{1}, C_{2}$ be the components of $\partial P$ indexed so that $C_{1} \cap H_{2}=\varnothing$ and $C_{2} \cap H_{1}=\varnothing$. Fix orientations on these curves using the following convention; $C_{1}$ is homotopic to $w_{1}, C_{2}$ is homotopic to $w_{2}$, and $C_{0}$ is homotopic to $w_{1} w_{2}$. For $i \in\{1,2\}$, choose points $x_{i}$ on $C_{i} \cap D$, and oriented arcs $\sigma_{i}$ in $D$ going from $x_{0}$ to $x_{i}$, with $\operatorname{int}\left(\sigma_{i}\right) \cap W=\varnothing$. Define a map $b:(P, \partial P) \rightarrow(P, \partial P)$ requiring that:

(1) $C_{0} \mapsto C_{2}$ by an orientation preserving homeomorphism,

(2) $C_{1} \mapsto C_{2}$ by an o.p. homeomorphism which takes $x_{1}$ to $x_{2}$,

(3) $C_{2} \mapsto\left\{x_{2}\right\}$,

(4) $x_{0} \mapsto x_{0}, \sigma_{1} \mapsto w_{1} w_{2}^{-1} w_{1} \sigma_{2}$ and $\sigma_{2} \mapsto \sigma_{2}$.

It is easy to check that (1)-(4) can be extended to a boundarypreserving self-map of $P$. On the level of integral homology the endomorphism $b_{*}$ of $H_{1}(P ; Z)$ sends $w_{1} \mapsto w_{2}$ and $w_{2} \mapsto 1$. Thus the Lefschetz number $L(b)$ is equal to one. Furthermore, it is shown in case (iii) of the proof of Theorem 4.10 in [BS], that $N_{\partial}(b)=1$. On the contrary we show that

THEOREM 4.3. $M F_{\partial}[b]=2$ and hence, the pants surface is not boundary-Wecken. 
Proof. Let $h$ be a fixed point minimizing map which is homotopic to $b$ through a boundary-preserving homotopy, and such that $h^{-1}(A)$ is a 1-dimensional proper submanifold of $P$ which does not contain any inessential simple closed curves. Without loss of generality, we assume that $h\left(C_{2}\right)=x_{2}$ and $h \mid C_{i}: C_{i} \rightarrow C_{2}, i \in\{0,1\}$, is a homeomorphism with the property that $h\left(C_{i} \backslash H_{1}\right) \subset\left(C_{2} \cap D\right)$, and $h\left(x_{1}\right)=x_{2}$. It is then clear that $h^{-1}(A)$ consists of two proper arcs each going from $C_{0}$ to $C_{1}$, together with a number of essential simple closed curves. Due to the presence of the arcs, the only possible simple closed curves are $w_{2}$-curves.

We now go back to constructions given in previous sections to get more control on the curves in $h^{-1}(A)$. First the collar construction (given in the proof of Lemma 2.1) can be used to produce a new map in the same boundary-preserving homotopy class as $b$ as follows: Add collars to each of $C_{0}$ and $C_{1}$ to obtain $P^{\prime}$. Extend the proper arcs of $h^{-1}(A)$ in the collars so that the new arcs in $P^{\prime}$ are isotopic (rel $\left.\partial P^{\prime}\right)$ to arcs contained in $H_{1}$. Now define $h$ on the new boundary components so that $h \mid C_{i}^{\prime}$ has the same properties as $h \mid C_{i}$. Extend to the rest of the collars by mapping into a neighborhood of $C_{2}$, and finish by adjusting the map on a neighborhood of $C_{0} \cup C_{1}$ so that these curves are now mapped into the interior of $P^{\prime}$. The fact that $h\left(C_{0} \cup C_{1}\right) \subset C_{2}$ ensures that when constructing this new map (which by abuse of notation we still call $h$ ) no fixed points are added. For the simple closed curves, condition (4) in the definition of $b$ implies that there must be at least six. If there are any more, the excess must be paired and so the annulus construction (in the proof of Proposition 3.2) can be used to remove them. According to the definition of $b$, the $\mu$-values of the six $w_{2}$-curves read $2,1,3,4,2,1$ in order along the arc $\sigma_{1}^{-1} \sigma_{2}$.

Let $g$ be a taut map with $g^{-1}(A)$ isotopic $\operatorname{rel} \partial P$ to $h^{-1}(A)$. By Theorem 4.2, we may assume that there is a merging sequence $g \stackrel{\Lambda}{\rightarrow} \hat{g}$ with $h=\hat{g}_{\text {min }}$. Let $R_{i}, i \in\{1,2\}$, be the component of $D \backslash g^{-1}(A)$ meeting $C_{i}$. By construction, $g\left(R_{i}\right) \subset D$ and so each is a critical region for $g$. Clearly, $R_{2}$ contains the fixed point $x_{2}$. We claim that $R_{1}$ must contain a fixed point as well. To see this, let $Y_{0}$ denote the $w_{2}$-curve furthest away from $C_{2}$ and let $Y$ denote the annulüs in $P$ which is bounded by $Y_{0}$ and $C_{2}$. Thus, $Y$ contains all of the $w_{2}$-curves in $g^{-1}(A)$. Let $g^{\prime}$ be a non-boundary-preserving map which agrees with $g$ on $P \backslash Y$, and which maps all of $Y$ into $A_{2}$. Then $g^{\prime}$ is homotopic to $g$ and thus $L\left(g^{\prime}\right)=1$. The claim is now 
established by noting that $g^{\prime}$ has no fixed points in $P \backslash R_{1}$. Hence index $\left(R_{1}\right)=L\left(g^{\prime}\right)=1$.

It is easy to see that $P \backslash\left(A \cup g^{-1}(A)\right)$ has a total of 17 components; 3 in $H_{1}, 7$ in $D, 7$ in $H_{2}$. Of these, exactly five are critical regions for $g$. Aside from $R_{1}$ and $R_{2}$, each of the others is contained in the interior of $Y$ and is bounded by subarcs from two $w_{2}$-curves, a subarc of $A_{3}$ and a subarc of $A_{4}$. Suppose that $\lambda$ is a merging arc for $g$. If $\lambda_{0}$ is a component of $\lambda \backslash A$, then condition (a3) says that $\lambda_{0}$ meets a $w_{2}$-curve only when one of its endpoints is also an endpoint of $\lambda$ and the other is contained in $A_{1} \cup A_{2}$. This, together with condition (a4), guarantees that none of the three critical regions in the interior of $Y$ contains an endpoint of $\lambda$. Thus, the endpoints of $\lambda$ must be $x_{2}$ and the fixed point in $R_{1}$. Starting with the endpoint $x_{2}$, $\lambda$ must first traverse each of the six $w_{2}$-curves with $\mu$-values in the order $1,2,4,3,1,2$. Thus $g(\lambda)$ must contain a subarc starting at $x_{2}$ and traversing $A$ in the order $A_{1}, A_{2}, A_{4}, A_{3}, A_{1}, A_{2}$ and hence, $\lambda$ must contain such a subarc as well. But this is a contradiction, as any curve starting at $x_{2}$ and traversing $A$ in that order must have self crossings. Thus, $\Lambda$ must be empty and $\hat{g}=g$. By Lemma 2.4 , the critical region for $h$ corresponding to $R_{1}$ has nonzero index and so it must contain a fixed point which is in the interior $P$.

\section{REFERENCES}

[BS] R. F. Brown and B. J. Sanderson, Fixed points of boundary-preserving maps of surfaces, Pacific J. Math., 158 (1993), 243-264.

[J] B. J. Jiang, Fixed points and braids, II, Math. Ann., 272 (1985), 249-256.

[K1] M. R. Kelly, Minimizing the number of fixed points for self-maps of compact surfaces, Pacific J. Math., 126 (1987), 81-123.

[K2] _ Minimizing the cardinality of the fixed point set for self-maps of surfaces with boundary, Michigan Math. J., 39 (1992), 201-217.

[S] H. Schirmer, A relative Nielsen number, Pacific J. Math., 118 (1986), 459-473.

[W] F. Wecken, Fixpunktklassen, III, Math. Ann., 118 (1942), 544-577.

Received November 3, 1991 and in revised form, July 18, 1992.

LOYOLA UNIVERSITY

New ORLEANS, LA 70118 



\title{
PACIFIC JOURNAL OF MATHEMATICS
}

Founded by

\author{
E. F. BECKENBACH (1906-1982) F. WolF (1904-1989)
}

\section{EDITORS}

Sun-Yung A. Chang

(Managing Editor)

University of California

Los Angeles, CA 90024-1555

chang@math.ucla.edu

\section{F. Michael Christ}

University of California

Los Angeles, CA 90024-1555

christ@math.ucla.edu

HeRbert Clemens

University of Utah

Salt Lake City, UT 84112

clemens@math.utah.edu
Thomas ENRIGHT

University of California, San Diego

La Jolla, CA 92093

tenright@ucsd.edu

Nicholas ERCOLANI

University of Arizona

Tucson, AZ 85721

ercolani@math.arizona.edu

\section{R. FINN}

Stanford University

Stanford, CA 94305

finn@gauss.stanford.edu

VAughan F. R. Jones

University of California

Berkeley, CA 94720

vfr@math.berkeley.edu
SteVen KerckhofF

Stanford University

Stanford, CA 94305

spk@gauss.stanford.edu

Martin ScharlemanN University of California Santa Barbara, CA 93106 mgscharl@henri.ucsb.edu

\section{Harold Stark}

University of California, San Diego La Jolla, CA 92093

V. S. VARADARAJAN University of California Los Angeles, CA 90024-1555 vsv@math.ucla.edu

\section{SUPPORTING INSTITUTIONS}

UNIVERSITY OF ARIZONA

UNIVERSITY OF BRITISH COLUMBIA

CALIFORNIA INSTITUTE OF TECHNOLOGY

UNIVERSITY OF CALIFORNIA

UNIVERSITY OF MONTANA

UNIVERSITY OF NEVADA, RENO

NEW MEXICO STATE UNIVERSITY

OREGON STATE UNIVERSITY
UNIVERSITY OF OREGON

UNIVERSITY OF SOUTHERN CALIFORNIA

STANFORD UNIVERSITY

UNIVERSITY OF HAWAII

UNIVERSITY OF UTAH

WASHINGTON STATE UNIVERSITY

UNIVERSITY OF WASHINGTON 


\section{PACIFIC JOURNAL OF MATHEMATICS}

Volume $161 \quad$ No. $1 \quad$ November 1993

Tangential and normal Euler numbers, complex points, and

singularities of projections for oriented surfaces in four-space

ThOMAS FRANCIS BANCHOFF and FrANK ALBERT FARRIS

Studying links via closed braids. III. Classifying links which are closed 3-braids

JOAN Birman and William W. MEnAsCo

Dehn functions of groups and extensions of complexes

STEPHEN GARY BRICK

Contact structures on $(n-1)$-connected $(2 n+1)$-manifolds

\section{HANSJÖRG GEIGES}

The relative Nielsen number and boundary-preserving surface maps

MiCHAEL R. KELLY

Besov spaces, mean oscillation, and generalized Hankel operators

\section{MARCo Maria PELOSO}

On the nonoccurrence of the Coxeter graphs $\beta_{2 n+1}, D_{2 n+1}$ and $E_{7}$ as 185 the principal graph of an inclusion of $\mathrm{II}_{1}$ factors

ViakALATHUR SHANKAR SUNDER and A. K. ViJAYARAJAN 